\title{
SIMULATION OF A PERIODIC DIELECTRIC CORRUGATION WITH AN EQUIVALENT ANISOTROPIC LAYER
}

\author{
Kamal Sarabandi \\ Department of Electrical Engineering and Computer Science \\ The University of Michigan \\ Ann Arbor, Michigan 48109-2122 \\ Received September 7, 1990
}

\begin{abstract}
A periodic, corrugated, dielectric layer is simulated by an anisotropic dielectric layer of equal thickness. The tensor elements of the equivalent dielectric layer are given in terms of the permittivity of the dielectric material, the period of the surface corrugation, and the width of the corrugations. The validity of this technique is verified by comparing the reflection coefficient of the equivalent layer with that of the corresponding corrugated layer using the moment method. Employing a multiple layer approach, the technique is extended to handle periodic surfaces with arbitrary cross sections which can be used to design millimeter wave dielectric plate polarizers and absorbers.
\end{abstract}

\section{Introduction}

The purpose of this paper is to simulate a periodic corrugated dielectric layer by a homogeneous anisotropic layer. Figure 1 shows the geometry of a corrugated surface over a layered medium. If we let the top $(y=t)$ and the bottom $(y=0)$ interfaces approach, respectively, $+\infty$ and $-\infty$, a medium of periodic slabs as shown in Fig. 2 will be created. Thus the periodic dielectric corrugation can be viewed as a layer of a medium comprised of homogeneous dielectric slabs of identical material which are equally spaced. Ignoring the effect of discontinuities, the problem is 
reduced to finding the equivalent dielectric tensor of the periodic slab medium.

Depending on the polarization of the fields and the boundary conditions imposed, a variety of different modes can be supported by this structure. Modeling of grooved-dielectric surfaces with anisotropic homogeneous media was first studied, to our knowledge, by Morita and Cohen [3]. They analyzed this problem by simulating the periodic slab medium using a partially-filled waveguide, then by solving the appropriate transcedental equation the propagation constant in the corrugation was obtained. These results are used in many problems such as matching dielectric lens surfaces [3] and designing broadband radomes $[1,4]$.

Since the interest is in the modes that are excited by an incident plane wave, simulating the array by a partially-filled waveguide, which forces the tangential electric field to be zero at the waveguide walls,

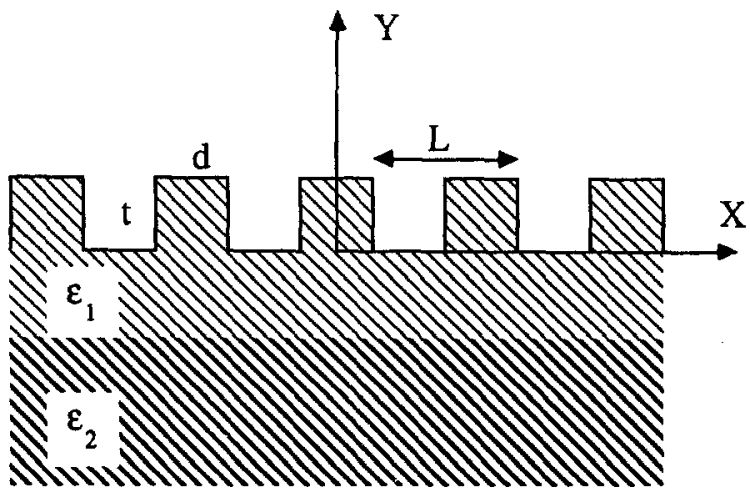

Figure 1: Geometry of the corrugated surface.

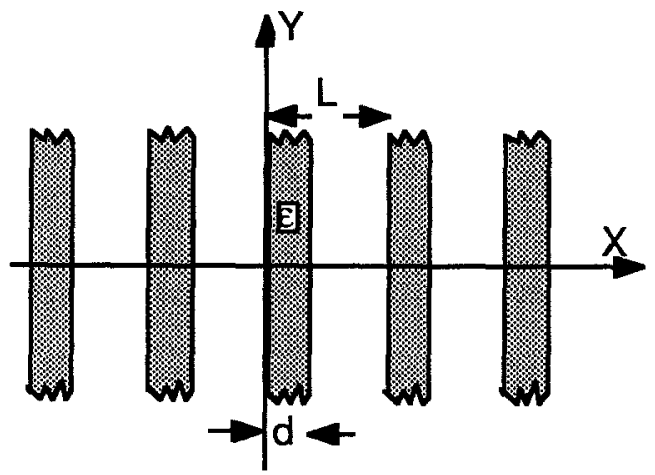

Figure 2: An array of infinite dielectric slabs. 
may not be appropriate. Here we impose a condition that supports the modes which would be excited by a plane wave incident on the structure. This approach will provide a formula for the elements of the equivalent dielectric tensor which is different from that reported by Morita and Cohen. The validity of this technique is verified in Section 5 by a numerical solution of corrugated surfaces. In Section 4 the reflection coefficient of uniaxial layered media is derived to extend the applicabilities of the equivalent dielectric technique to periodic surfaces with arbitrary cross sections.

\section{Theoretical Analysis}

To proceed with the analysis, suppose that similar dielectric slabs of an infinite array with thickness $d$ and dielectric constant $\epsilon$ are parallel to the $y-z$ plane. Further assume that the period of the structure is denoted by $L$. The geometry of the problem is depicted in Fig. 2. If the $x-y$ plane is the plane of incidence, the solution is independent of $z$ and therefore the waves can be separated into $\mathrm{E}$ - and $\mathrm{H}$-polarized waves. Each period of the medium can be divided into two regions and, depending on the polarization, the $z$ component of the electric or magnetic field must satisfy the wave equation, i.e.

$$
\begin{array}{ll}
\left(\frac{\partial^{2}}{\partial x^{2}}+\frac{\partial^{2}}{\partial y^{2}}+\epsilon k_{0}^{2}\right) \Psi^{I}(x, y)=0 & 0 \leq x \leq d \\
\left(\frac{\partial^{2}}{\partial x^{2}}+\frac{\partial^{2}}{\partial y^{2}}+k_{0}^{2}\right) \Psi^{I I}(x, y)=0 & d \leq x \leq L
\end{array}
$$

where $\Psi(x, y)=E_{z}(x, y)$ or $H_{z}(x, y)$. Using separation of variables and requiring the phase matching condition, the solutions of (1) and (2) take the following forms

$$
\begin{gathered}
\Psi^{I}(x, y)=\left[A e^{i k_{x}^{I} x}+B e^{-i k_{x}^{I} x}\right] e^{i k_{y} y} \\
\Psi^{I I}(x, y)=\left[C e^{i k_{x}^{I I} x}+D e^{-i k_{x}^{I I} x}\right] e^{i k_{y} y}
\end{gathered}
$$

where $k_{y}$ is the propagation constant in the periodic medium which must satisfy

$$
\begin{aligned}
& \left(k_{x}^{I}\right)^{2}+k_{y}^{2}=\epsilon k_{0}^{2} \\
& \left(k_{x}^{I I}\right)^{2}+k_{y}^{2}=k_{0}^{2}
\end{aligned}
$$

In an attempt to find the unknown coefficients, we use the condition 
that the tangential components of the electric and magnetic fields must be continuous at $x=d$, which constitutes two equations. Two more equations can be obtained by applying Floquet's theorem for periodic differential equations which requires $\Psi(x+L, y)=\sigma \Psi(x, y)$ for some constant $\sigma$. To set an appropriate value for $\sigma$, suppose the medium is simulated as an equivalent homogeneous dielectric. If a plane wave illuminates the half-space of the equivalent homogeneous medium at an angle $\phi_{0}$, the $x$ dependency would be of the form $e^{i k_{0} \sin \phi_{0} x}$. This dependency suggests that we need to impose a progressive phase condition, i.e. let $\sigma=e^{i k_{0} \sin \phi_{0} L}$. Therefore the other two equations become

$$
\begin{gathered}
E_{\tan }(0+L, y)=e^{i k_{0} \sin \phi_{0} L} E_{\tan }(0, y) \\
H_{\tan }(0+L, y)=e^{i k_{0} \sin \phi_{0} L} H_{\tan }(0, y) .
\end{gathered}
$$

Application of the mentioned boundary conditions for the E polarization gives the following equations

$$
\begin{aligned}
& A e^{i k_{x}^{I} d}+B e^{-i k_{x}^{I} d}=C e^{i k_{x}^{I I} d}+D e^{-i k_{x}^{I I} d} \\
& k_{x}^{I}\left[A e^{i k_{x}^{I} d}-B e^{-i k_{x}^{I} d}\right]=k_{x}^{I I}\left[C e^{i k_{x}^{I I} d}-D e^{-i k_{x}^{I I} d}\right] \\
& A+B=\left[C e^{i k_{x}^{I I} l}+D e^{-i k_{x}^{I I} I}\right] e^{-i k_{0} \sin \phi_{0} L} \\
& k_{x}^{I}[A-B]=k_{x}^{I I}\left[C e^{i k_{x}^{I I} l}-D e^{-i k_{x}^{I I} l}\right] e^{-i k_{0} \sin \phi_{0} L}
\end{aligned}
$$

Since we are interested in the nontrivial solution of the above linear equations the determinant of the coefficient matrix must be set to zero. This condition provides an equation for $k_{x}^{I}$ and $k_{x}^{I I}$ and is given by

$$
\begin{gathered}
-\left(\frac{k_{x}^{I}}{k_{x}^{I I}}+\frac{k_{x}^{I I}}{k_{x}^{I}}\right) \sin \left(k_{x}^{I} d\right) \sin \left(k_{x}^{I I}(L-d)\right)+2 \cos \left(k_{x}^{I} d\right) \cos \left(k_{x}^{I I}(L-d)\right)= \\
2 \cos \left(k_{0} \sin \phi_{0} L\right)
\end{gathered}
$$

Dispersion relations (5) and (6) give rise to additional equations for $k_{x}^{I}$ and $k_{x}^{I I}$; i.e.,

$$
\left(k_{x}^{I}\right)^{2}-\left(k_{x}^{I I}\right)^{2}=k_{0}^{2}(\epsilon-1)
$$

The transcedental equation (10) together with (11) can be solved simultaneously to find the propagation constants. It is worth noting that in the limiting case when the periodic medium approaches a homogeneous one, then $k_{x}^{I} \rightarrow k_{x}^{I I}$ and (10) reduces to

$$
\cos \left(k_{x} L\right)=\cos \left(k_{0} \sin \phi_{0} L\right)
$$

which implies $k_{x}=k_{0} \sin \phi_{0}$, as expected.

After solving (10) and (11) for $k_{x}^{I I}$, the propagation constant in the 
$y$-direction can be obtained from

$$
k_{y}^{2}=k_{0}^{2}-\left(k_{x}^{I I}\right)^{2}
$$

It should be pointed out that the solution for $k_{x}^{I I}$ is not unique and therefore this structure can support many modes corresponding to different values of $k_{x}^{I I}$. The dominant mode for this structure corresponds to a value of $k_{x}^{I I}$ such that the imaginary part of $k_{y}$ is minimum. For the equivalent homogeneous medium with permittivity $\epsilon_{z}$, the propagation constant in the $y$-direction would be

$$
k_{y}^{2}=k_{0}^{2}\left(\epsilon_{z}-\sin ^{2} \phi_{0}\right)
$$

Comparing (12) and (13) it can be deduced that

$$
\epsilon_{z}=\sin ^{2} \phi_{0}+1-\left(\frac{k_{x}^{I I}}{k_{0}}\right)^{2}
$$

From the symmetry of the problem it is obvious that if the electric field is in the $y$-direction the equivalent dielectric would be the same, that is $\epsilon_{y}=\epsilon_{z}$.

Using a similar procedure the following transcedental equation for $\mathrm{H}$ polarization can be obtained

$$
\begin{gathered}
-\left(\frac{\epsilon k_{x}^{I I}}{k_{x}^{I}}+\frac{k_{x}^{I}}{\epsilon k_{x}^{I I}}\right) \sin \left(k_{x}^{I} d\right) \sin \left(k_{x}^{I I}(L-d)\right)+2 \cos \left(k_{x}^{I} d\right) \cos \left(k_{x}^{I I}(L-d)\right)= \\
2 \cos \left(k_{0} \sin \phi_{0} L\right)
\end{gathered}
$$

As will be shown later the dispersion relationship in this case is:

$$
k_{y}^{2}=k_{0}^{2} \frac{\epsilon_{x}}{\epsilon_{z}}\left(\epsilon_{z}-\sin ^{2} \phi_{0}\right)
$$

and the equivalent dielectric constant $\epsilon_{x}$ can be obtained from

$$
\epsilon_{x}=\frac{\epsilon_{z}}{\epsilon_{z}-\sin ^{2} \phi_{0}}\left[1-\left(\frac{k_{x}^{I I}}{k_{0}}\right)^{2}\right]
$$

where $k_{x}^{I I}$ is the solution of (15) and (11). Since $\epsilon_{x} \neq \epsilon_{y}=\epsilon_{z}$ the equivalent medium is uniaxial with the optical axis being parallel to the $x$-axis. 


\section{Low Frequency Approximation}

An analytical solution of equations (10) and (15) for $k_{x}^{I}$ and $k_{x}^{I I}$ cannot be derived, in general, but using Newton's or Muller's method numerical solutions can easily be obtained. One of the cases where approximate expressions for $\epsilon_{x}$ and $\epsilon_{y}=\epsilon_{z}$ can be derived is the low frequency regime where $L<0.2 \lambda_{0}$. In this approximation the sine and cosine functions are replaced with their Taylor series expansion keeping terms up to the quadratic term. Therefore equations (10) and (15), respectively, reduce to

$$
\left\{\begin{array}{l}
\left(k_{x}^{I}\right)^{2} d+\left(k_{x}^{I I}\right)^{2}(L-d)=\left(k_{0} \sin \phi_{0}\right)^{2} L \\
\left(k_{x}^{I}\right)^{2} \frac{d}{\epsilon}+\left(k_{x}^{I I}\right)^{2}(L-d)=\left(k_{0} \sin \phi_{0}\right)^{2} \frac{L^{2}}{\epsilon d+(L-d)}
\end{array}\right.
$$

These equations together with (11) can be solved easily to obtain

$$
\begin{aligned}
& \epsilon_{y}=\epsilon_{z}=1+(\epsilon-1) \frac{d}{L} \\
& \epsilon_{x}=\frac{\epsilon}{\epsilon(1-d / L)+d / L}
\end{aligned}
$$

Note that when $d \rightarrow L$, the elements of the dielectric tensor approach the permittivity of region $I$, i.e. $\epsilon_{x}, \epsilon_{y}, \epsilon_{z} \rightarrow \epsilon$ and when $d \rightarrow 0$, then $\epsilon_{x}, \epsilon_{y}, \epsilon_{z} \rightarrow 1$, as expected. At low frequencies, as shown by (18) and (19), the equivalent permittivities are neither functions of frequency $\left(k_{0}\right)$ nor functions of incidence angle.

\section{Reflection Coefficient of a Uniaxial Layered Medium}

Consider a multilayer dielectric half-space as shown in Fig. 3. Suppose each dielectric layer is uniaxial and the optical axes of all the layers are parallel to the $x$ axis. Further assume that a plane wave whose plane of incidence is parallel to the $x-y$ plane is illuminating the stratified medium from above at an angle $\phi_{0}$. The interface of the $n$th and the $(n+1)$ th layers is located at $y=d_{n}$.

The dielectric tensor of the $n$th layer is defined by $\mathbf{D}^{n}=\epsilon_{n} \mathbf{E}^{n}$ and is assumed to be of the following form 


$$
\epsilon_{n}=\epsilon_{0}\left[\begin{array}{ccc}
\epsilon_{x n} & 0 & 0 \\
0 & \epsilon_{n} & 0 \\
0 & 0 & \epsilon_{n}
\end{array}\right]
$$

and its permeability is that of free space $\left(\mu_{n}=\mu_{0}\right)$. In this situation two plane waves are generated in the dielectric slabs: one ordinary and one extraordinary. For the ordinary wave the electric field and the electric displacement are parallel and both are perpendicular to the principal plane (the plane parallel to the optical axis and the direction of propagation) [2], hence the magnetic field is in the $x$-direction (see Fig. 3). For the extraordinary wave, however, the electric displacement and the magnetic field lie in the principal plane and the $y$-z plane respectively which force the electric field to be parallel to the $x$-axis. Therefore the ordinary or the extraordinary waves can be generated, respectively, by a magnetic or an electric Hertz vector potential having only an $x$ component. For ordinary waves $\mathbf{D}^{n}=\epsilon_{0} \epsilon_{n} \mathbf{E}^{n}$ and the magnetic Hertz potential $\left(\boldsymbol{\Pi}_{m}^{n}=\Pi_{o}^{n} \hat{x}\right)$ must satisfy the wave equation, i.e.

$$
\nabla^{2} \Pi_{o}^{n}+k_{0}^{2} \epsilon_{n} \Pi_{o}^{n}=0
$$

The electric and magnetic fields in terms of the magnetic Hertz potential where there is no variation with respect to $z$ are given by

$$
\mathbf{E}_{o}^{n}=-i k_{0} Z_{0} \frac{\partial \Pi_{o}^{n}}{\partial y} \hat{z}
$$

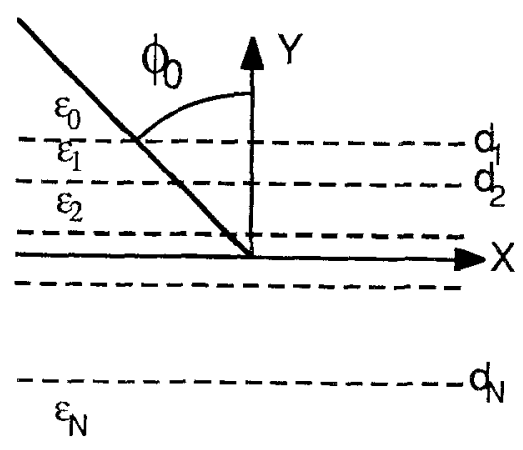

Figure 3: Plane wave reflection from a stratified uniaxial dielectric halfspace. 


$$
\mathbf{H}_{o}^{n}=-\frac{\partial^{2} \Pi_{o}^{n}}{\partial y^{2}} \hat{x}+\frac{\partial^{2} \Pi_{o}^{n}}{\partial x \partial y} \hat{y}
$$

The electric and magnetic fields associated with the extraordinary wave, as discussed earlier, can be derived from an electric Hertz vector potential, i.e.

$$
\mathbf{H}_{e}^{n}=-i k_{0} Y_{0} \nabla \times \Pi_{\mathrm{e}}^{n}
$$

which implies

$$
\mathbf{E}_{e}^{n}=k_{0}^{2} \boldsymbol{\Pi}_{e}^{n}+\nabla \Phi^{n}
$$

where $\Phi^{n}$ is an arbitrary scalar function. Using (20) the electric displacement in the $n$th layer is represented by

$$
\mathbf{D}_{e}^{n}=\epsilon_{0}\left[\left(\epsilon_{x n}-\epsilon_{n}\right) E_{e x}^{n} \hat{x}+\epsilon_{n} \mathbf{E}_{e}^{n}\right]
$$

Inserting (24) into (25) and noting that

$$
\mathrm{D}_{e}^{n}=\nabla \nabla \cdot \boldsymbol{\Pi}_{e}^{n}-\nabla^{2} \boldsymbol{\Pi}_{e}^{n}
$$

we get

$$
\nabla^{2} \boldsymbol{\Pi}_{e}^{n}+\epsilon_{x n} k_{0}^{2} \boldsymbol{\Pi}_{e}^{n}+\left(\epsilon_{x n}-\epsilon_{n}\right) \frac{\partial \Phi^{n}}{\partial x} \hat{x}+\nabla\left(\epsilon_{n} \Phi^{n}-\nabla \cdot \boldsymbol{\Pi}_{e}^{n}\right)=0
$$

So far no condition has been imposed on the scalar function $\Phi^{n}$. To simplify the differential equation (26) let $\nabla \cdot \boldsymbol{\Pi}_{e}^{n}=\epsilon_{n} \Phi^{n}$ be the gauge condition. Therefore the electric Hertz vector potential must satisfy

$$
\nabla^{2} \Pi_{e}^{n}+\epsilon_{x n} k_{0}^{2} \Pi_{e}^{n}+\frac{\epsilon_{x n}-\epsilon_{n}}{\epsilon_{n}} \frac{\partial^{2} \Pi_{e}^{n}}{\partial x^{2}}=0
$$

In the special case where $\partial / \partial z=0$ the magnetic and electric fields of the extraordinary waves can be obtained from

$$
\begin{gathered}
\mathbf{H}_{e}^{n}=i k_{0} Z_{0} \frac{\partial \Pi_{e}^{n}}{\partial y} \hat{z} \\
\mathbf{E}_{e}^{n}=\left(k_{0}^{2} \Pi_{e}^{n}+\frac{1}{\epsilon_{n}} \frac{\partial^{2} \Pi_{e}^{n}}{\partial x^{2}}\right) \hat{x}+\frac{1}{\epsilon_{n}} \frac{\partial^{2} \Pi_{e}^{n}}{\partial x \partial y} \hat{y}
\end{gathered}
$$


The solution of the differential equations (21) and (27) subject to plane wave incidence can be represented by $e^{i\left(k_{o x}^{n} x \pm k_{o y}^{n} y\right)}$ and $e^{i\left(k_{e x}^{n} x \pm k_{e y}^{n} y\right)}$ respectively and upon substitution of these solutions in the corresponding differential equations the following dispersion relationships are obtained

$$
\begin{gathered}
\left(k_{o x}^{n}\right)^{2}+\left(k_{o y}^{n}\right)^{2}=\epsilon_{n} k_{0}^{2} \\
\frac{1}{\epsilon_{n}}\left(k_{\text {ex }}^{n}\right)^{2}+\frac{1}{\epsilon_{x n}}\left(k_{\text {ey }}^{n}\right)^{2}=k_{0}^{2}
\end{gathered}
$$

Imposing the phase matching condition, i.e. $k_{o x}^{n}=k_{e x}^{n}=k_{0} \sin \phi_{0}$, the dispersion relations simplify to

$$
k_{o y}^{n}=k_{0} \sqrt{\epsilon_{n}-\sin \phi_{0}}, \quad k_{e y}^{n}=k_{0} \sqrt{\frac{\epsilon_{x n}}{\epsilon_{n}}} \sqrt{\epsilon_{n}-\sin ^{2} \phi_{0}}
$$

The Hertz vector potentials in the $n$th layer can be written as

$$
\begin{aligned}
& \Pi_{o}^{n}=\left[A_{i}^{n} e^{-i k_{o y}^{n} y}+A_{r}^{n} e^{i k_{o y}^{n} y}\right] e^{i k_{0} \sin \phi_{0} x} \\
& \Pi_{e}^{n}=\left[B_{i}^{n} e^{-i k_{e y}^{n} y}+B_{r}^{n} e^{i k_{e y}^{n} y}\right] e^{i k_{0} \sin \phi_{0} x}
\end{aligned}
$$

In (32)-(33) the subscripts $i$ and $r$ in the coefficients denote the propagation in the negative and positive $y$ direction respectively.

Since there is no variation with respect to $z$, the incident wave can be decomposed into parallel $(\mathrm{H})$ and perpendicular $(\mathrm{E})$ polarization which would excite extraordinary and ordinary waves respectively. For $\mathrm{E}$ polarization the electric and magnetic fields in each region can be obtained from (22) and (23) using (32). In region $0, A_{i}^{0}$ is proportional to the incident amplitude, and $-A_{r}^{0} / A_{i}^{0}=R_{E}$ is the total reflection coefficient. In region $N+1$, which is semi-infinite, $A_{r}^{N+1}=0$. Imposing the boundary conditions, which requires continuity of tangential electric and magnetic fields at each dielectric interface, we can relate the field amplitudes in the $n$th region to those of $(n+1)$ th region. After some algebraic manipulation, the following recursive relationship can be obtained

$$
-\frac{A_{r}^{n}}{A_{i}^{n}}=\frac{\left(-A_{r}^{n+1} / A_{i}^{n+1}\right)+\Gamma_{o}^{n} e^{-i 2 k_{o y}^{n+1} d_{n+1}}}{\left(-A_{r}^{n+1} / A_{i}^{n+1}\right) \Gamma_{o}^{n}+e^{-i 2 k_{o y}^{n+1} d_{n+1}}} e^{-i 2 k_{o y}^{n} d_{n+1}}
$$

where 


$$
\Gamma_{o}^{n}=\frac{\sqrt{\epsilon_{n}-\sin ^{2} \phi}-\sqrt{\epsilon_{n+1}-\sin ^{2} \phi}}{\sqrt{\epsilon_{n}-\sin ^{2} \phi}+\sqrt{\epsilon_{n+1}-\sin ^{2} \phi}}
$$

Starting from $-A_{r}^{N+1} / A_{i}^{N+1}=0$ and using (34) repeatedly $R_{E}$ can be found.

For $\mathrm{H}$ polarization incidence the extraordinary waves are excited and using (33) in (28) and (29) the electric and magnetic fields in each region can be obtained. Following a similar procedure outlined for the $\mathrm{E}$ polarization case an identical recursive formula as given by (34) can be derived. The only difference is that $A$ is replaced by $B, k_{o y}$ by $k_{e y}$ and $\Gamma_{o}^{n}$ becomes $\Gamma_{e}^{n}$ which is given by

$$
\Gamma_{e}^{n}=\frac{\sqrt{\epsilon_{x(n+1)} \epsilon_{n+1}} \sqrt{\epsilon_{n}-\sin ^{2} \phi}-\sqrt{\epsilon_{x n} \epsilon_{n}} \sqrt{\epsilon_{n+1}-\sin ^{2} \phi}}{\sqrt{\epsilon_{x(n+1)} \epsilon_{n+1}} \sqrt{\epsilon_{n}-\sin ^{2} \phi}+\sqrt{\epsilon_{x n} \epsilon_{n}} \sqrt{\epsilon_{n+1}-\sin ^{2} \phi}}
$$

\section{Numerical Examples}

As mentioned earlier (10) and (15) can be solved using numerical methods. Here we use Newton's method to find the zeroes of the functions given by (10) and (15) in which $k_{x}^{I}$ is expressed in terms of $k_{x}^{I I}$ using (11). Before searching for zeroes we note that these functions are odd functions of $k_{x}^{I I}$, that is, if $\kappa$ is a solution so would be $-\kappa$ and both would give identical solutions for the equivalent permittivity as given by (14).

To study the behavior of the equivalent dielectric tensor elements, we consider a medium with $\epsilon=4+i 1$ and $L=\lambda_{0} / 4$. Figure 4 and 5 depict the variation of the real and imaginary parts of $\epsilon_{x}(\mathrm{H}$ polarization) and $\epsilon_{y}=\epsilon_{z}$ (E polarization) versus angle of incidence for $d / L=0.5$. It is shown that the dependence on incidence angle is very small and these results are in agreement with (18) and (19) within $10 \%$. The real and imaginary parts of the equivalent permittivities as a function of $d / L$ for incidence angle $\phi_{0}=45^{\circ}$ are shown in Figs. 6 and 7 respectively.

As $L / \lambda_{0}$ increases, the propagation loss factors $\left(\operatorname{Im}\left[k_{y}\right]\right)$ of different modes become comparable to each other. For example, if $L / \lambda_{0}=m / 2$, where $m$ is an integer, there would be at least two modes with equal propagation loss factor. Figures $8 \mathrm{a}$ through $8 \mathrm{c}$ show the location of zeroes of $(10)$ in $k_{x}^{I I}$-plane for $d / L=0.5, \phi_{0}=45^{\circ}$, and four values of $L / \lambda_{0}$. The real and imaginary parts of the equivalent permittivities as a function of $L / \lambda_{0}$ for $d / L=0.5$ and $\phi_{0}=45^{\circ}$ are shown in Figs. 


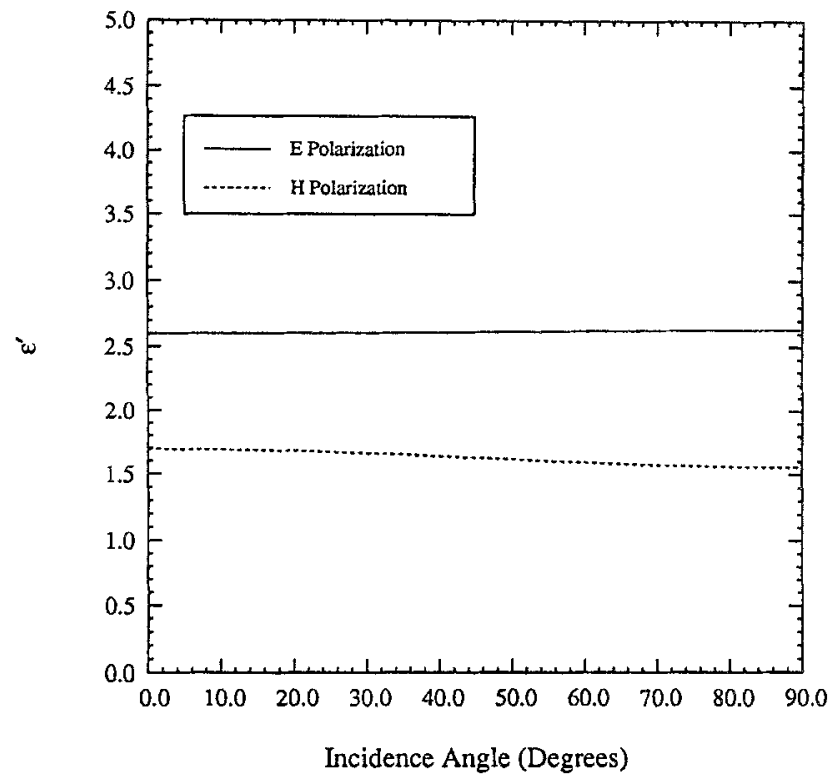

Figure 4: Real part of the equivalent dielectric tensor elements for periodic slab medium with $L=\lambda_{0} / 4, \epsilon=4+i 1$, and $d / L=0.5$ versus incidence angle; $\epsilon_{x}$ (H polarization), $\epsilon_{y}=\epsilon_{z}$ (E polarization).

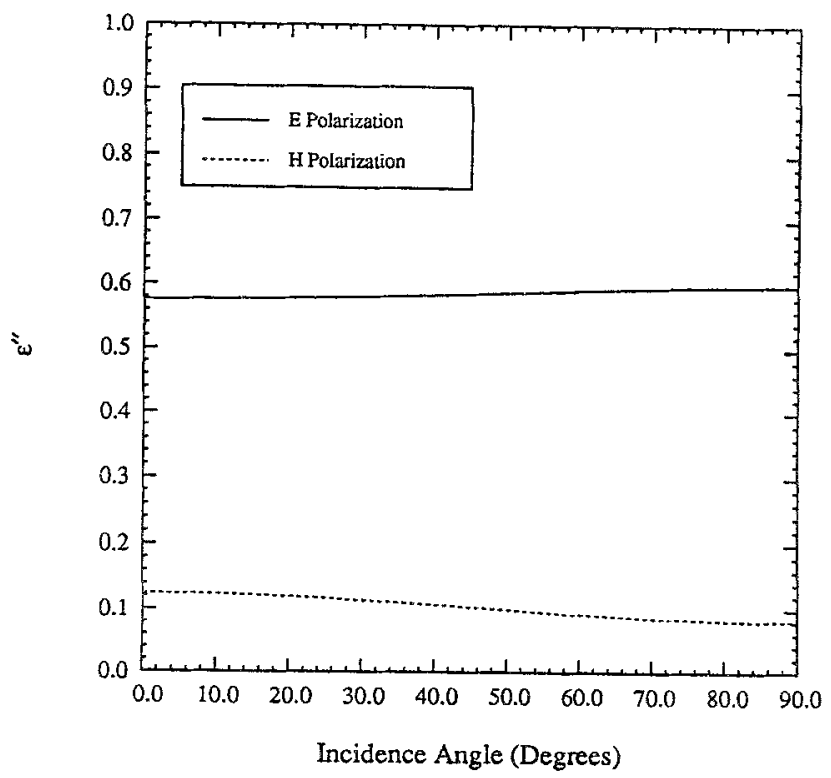

Figure 5: Imaginary part of the equivalent dielectric tensor elements for periodic slab medium with $L=\lambda_{0} / 4, \epsilon=4+i 1$, and $d / L=0.5$ versus incidence angle; $\epsilon_{x}$ (H polarization), $\epsilon_{y}=\epsilon_{z}$ (E polarization). 


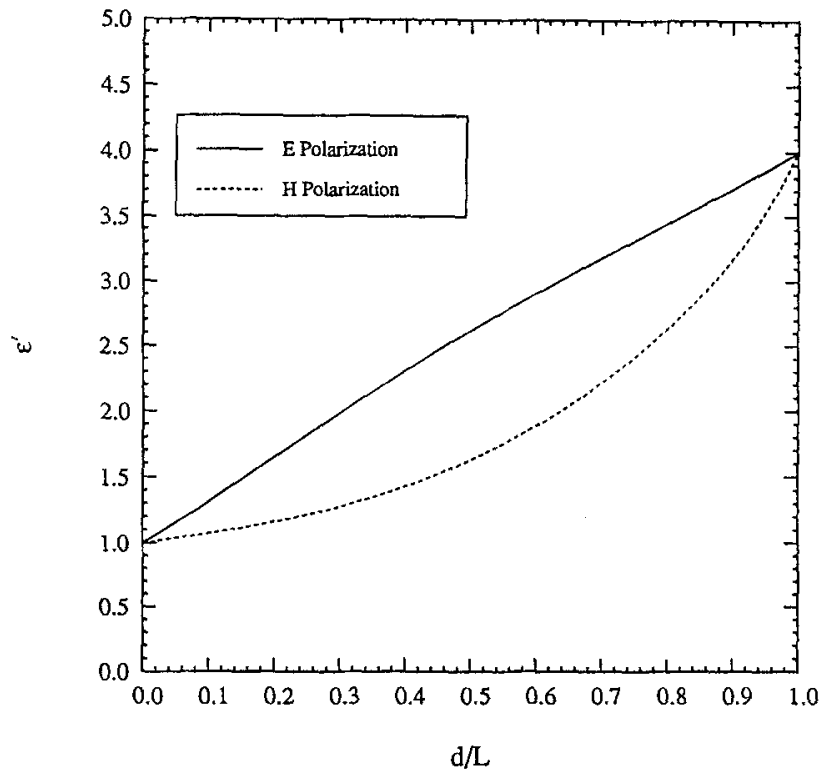

Figure 6: Real part of the equivalent dielectric tensor elements for a periodic slab medium with $L=\lambda_{0} / 4, \epsilon=4+i 1$, and $\phi_{0}=45^{\circ}$ versus $d / L ; \epsilon_{x}$ (H polarization), $\epsilon_{y}=\epsilon_{z}$ (E polarization).

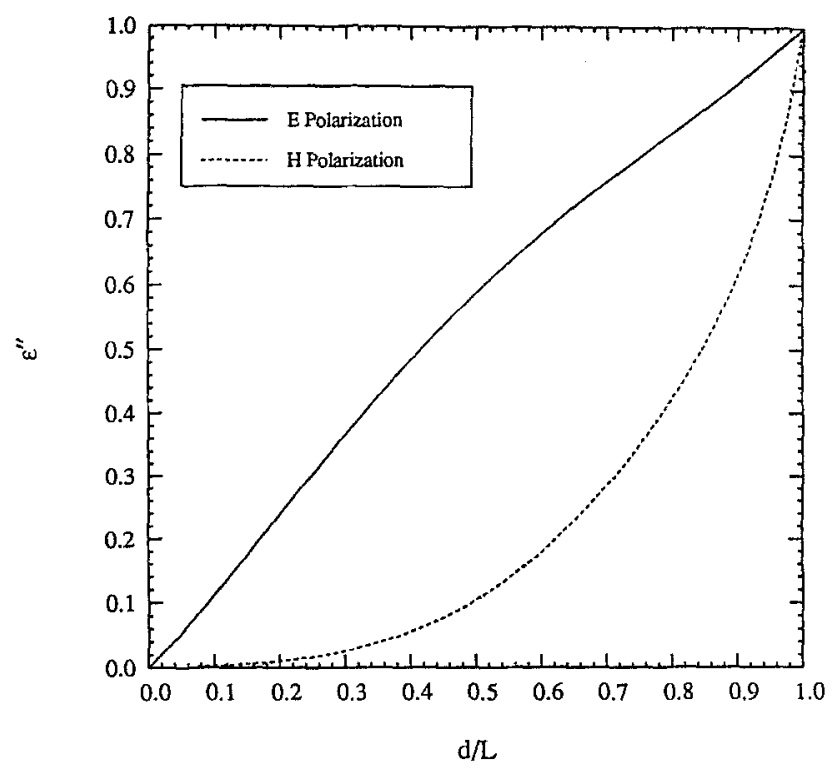

Figure 7: Imaginary part of the equivalent dielectric tensor elements for periodic siab medium with $L=\lambda_{0} / 4, \epsilon=4+i 1$, and $\phi_{0}=45^{\circ}$ versus $d / L ; \epsilon_{x}(\mathrm{H}$ polarization $), \epsilon_{y}=\epsilon_{z}$ (E polarization). 
(a)

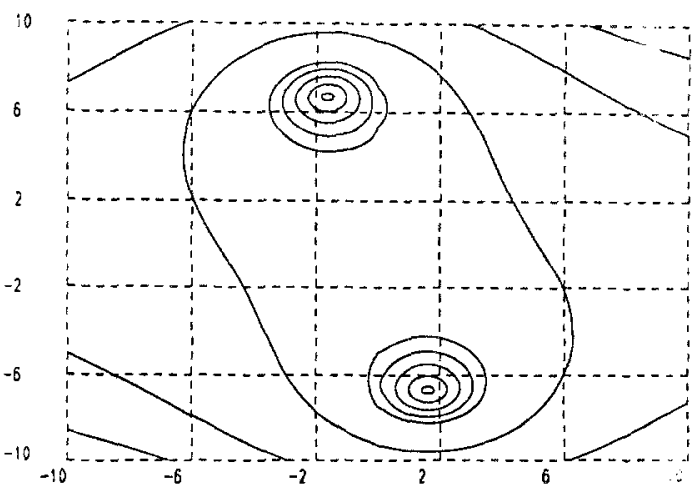

(b)

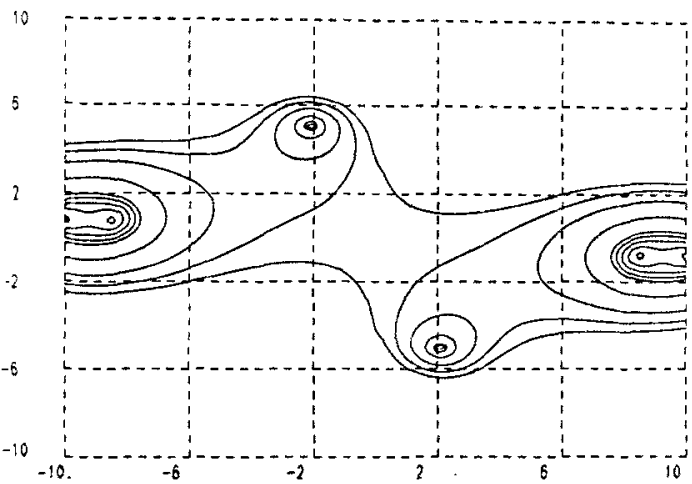

(c)

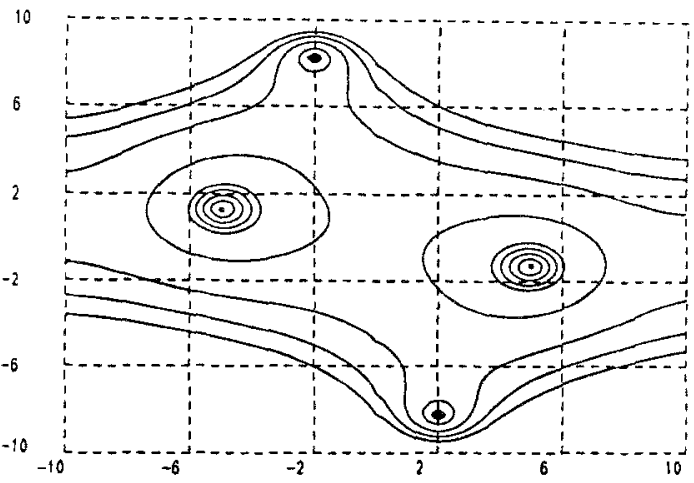

Figure 8: Location of zeros of (A-10)in the $k_{x}^{I I}$-plane for for the periodic slab medium with $\epsilon=4+i 1, \phi_{0}=45^{\circ}, d / L=0.5$, and (a) $L=0.2 \lambda_{0}$, (b) $L=0.5 \lambda_{0}$, (c) $L=0.8 \lambda_{0}$. 
9 and 10. The discontinuity in the equivalent permittivities at integer multiples of $\lambda_{0} / 2$ are due to the abrupt changes in the location of the zeroes in the $k_{x}^{I I}$-plane which correspond to the dominant mode.

To check the validity and applicability of this technique we compare the reflection coefficient of a corrugated surface using the moment method [5] with the reflection coefficient of the equivalent anisotropic medium as derived in Section 4. The geometry of the scattering problem is shown in Fig. 1. We consider a case where $L=\lambda_{0} / 4, d=t=\lambda_{0} / 8$, $\epsilon_{1}=4+i 1$, and $\epsilon_{2}=15+i 7$. The tensor elements for these parameters are found to be $\epsilon_{x}=1.65+i 0.12, \epsilon_{y}=\epsilon_{z}=2.6+i 0.58$. Figures 11 and 12 , respectively, compare the amplitude and phase of the reflection coefficients for both $\mathrm{E}$ and $\mathrm{H}$ polarizations. Excellent agreement between the results based on the moment method and the equivalent layer is an indication for the validity of the model. As frequency increases, because of presence of higher order modes, the discrepancy between the moment method and the equivalent layer results becomes more evident. Figures 13 and 14 show the reflection coefficient of a corrugated layer with $L / \lambda_{0}=0.4, d=t=0.2 \lambda, \epsilon_{1}=4+i 1$, and $\epsilon_{2}=15+i 7$. In this case $\epsilon_{x}=1.81+i 0.2, \epsilon_{y}=\epsilon_{z}=2.77+i 0.78$ and the agreement is still very good, but for $L>\lambda_{0} / 2$ where more than one Bragg mode exists the model fails to predict the reflection coefficient accurately.

Success of the equivalent layer in modeling rectangular corrugations can be extended to arbitrary periodic geometries. By approximating the cross section of the periodic surface with staggered increments of equal height, the surface can be viewed as a stack of corrugated layers (see Fig. 15). The height of each layer $\Delta H$ must be chosen such that $\Delta H \leq \lambda / 10$ where $\lambda$ is the wavelength in the material. Then each corrugated layer can be modeled by an equivalent anisotropic slab and the reflection coefficient of the resultant uniaxial layered medium can be obtained. To demonstrate this method consider a wedge-shape microwave absorber with permittivity $\epsilon=2+i 0.5$, period $L=0.4 \lambda_{0}$, wedge height $H=1.5 \lambda_{0}$, and base height $D=1 \lambda_{0}$. The number of layers considered here is 30 and the corresponding reflection coefficients for both $\mathrm{E}$ and $\mathrm{H}$ polarizations as a function of incidence angle is depicted in Fig. 16.

\section{Conclusions}

The equivalent dielectric tensor elements of a periodic array of infinite slabs is derived by requiring the phase of the Floquet's constant $(\sigma)$ to be the phase variation of an incoming plane wave over one period. It is 


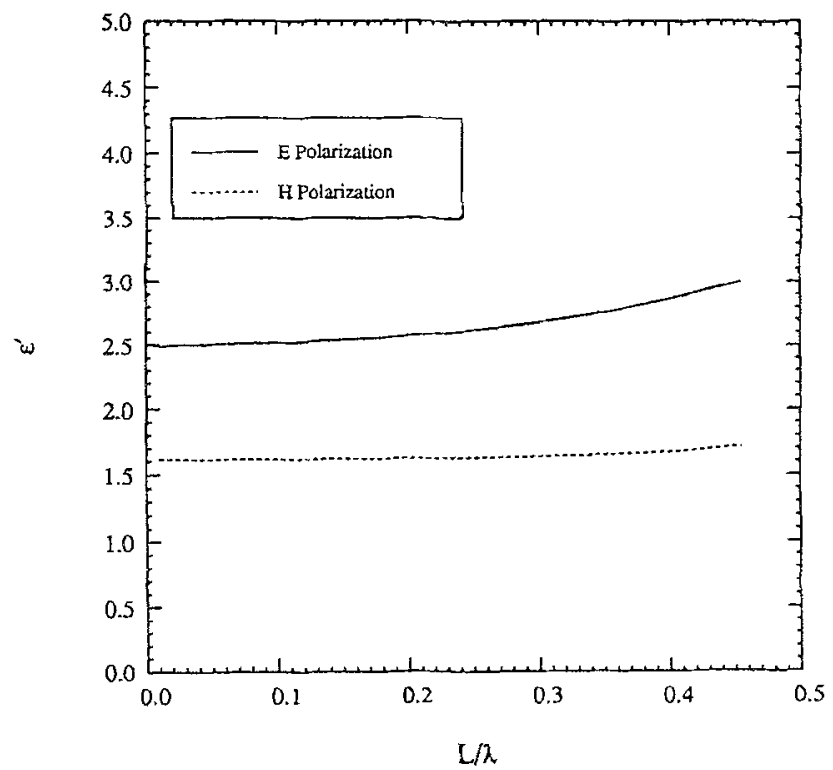

Figure 9: Real part of the equivalent dielectric tensor elements for periodic slab medium with $\epsilon=4+i 1$, and $\phi_{0}=45^{\circ}$, and $d / L=0,5$ versus $L / \lambda_{0} ; \epsilon_{x}$ (H polarization), $\epsilon_{y}=\epsilon_{z}$ (E polarization).

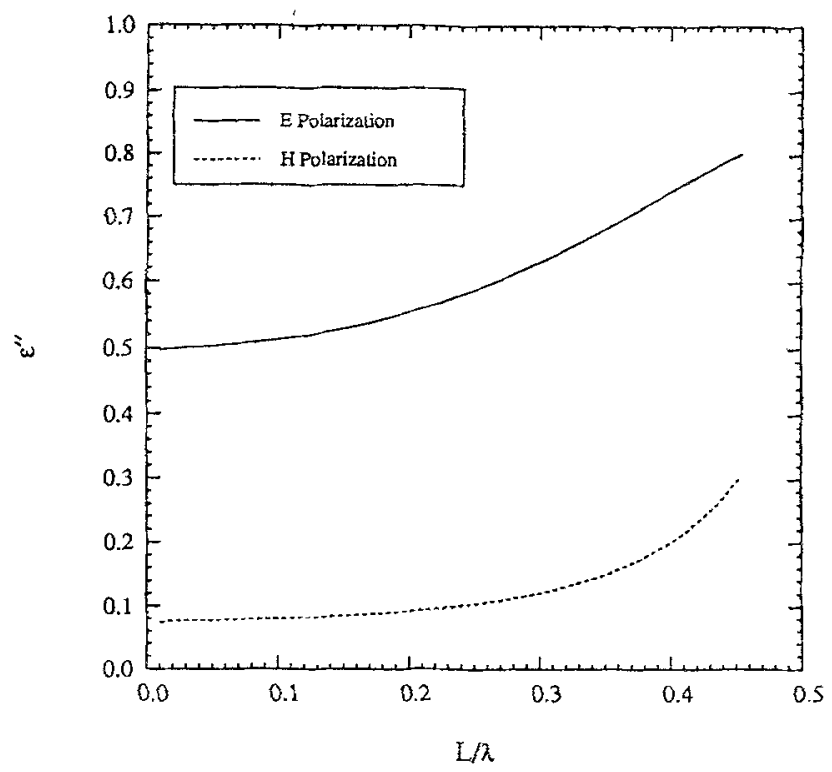

Figure 10: Imaginary part of the equivalent dielectric tensor elements for periodic slab medium with $\epsilon=4+i 1$, and $\phi_{0}=45^{\circ}$, and $d / L=0,5$ versus $L / \lambda_{0} ; \epsilon_{x}$ (H polarization), $\epsilon_{y}=\epsilon_{z}$ (E polarization). 


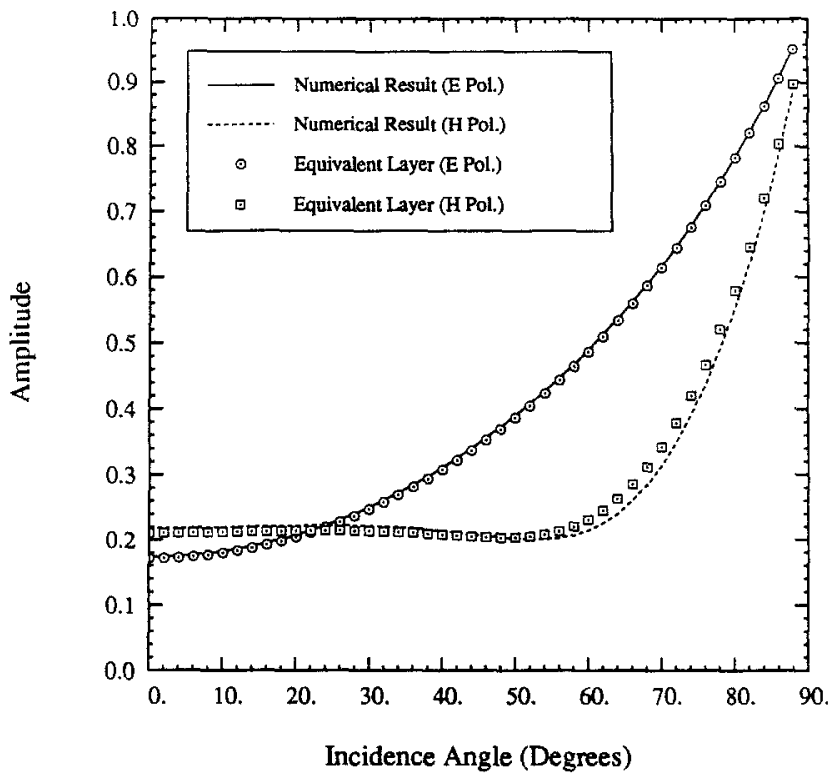

Figure 11: Amplitude of reflection coefficient of a corrugated surface for both $\mathrm{E}$ and $\mathrm{H}$ polarizations versus incidence angle; $L=0.25 \lambda_{0}$.

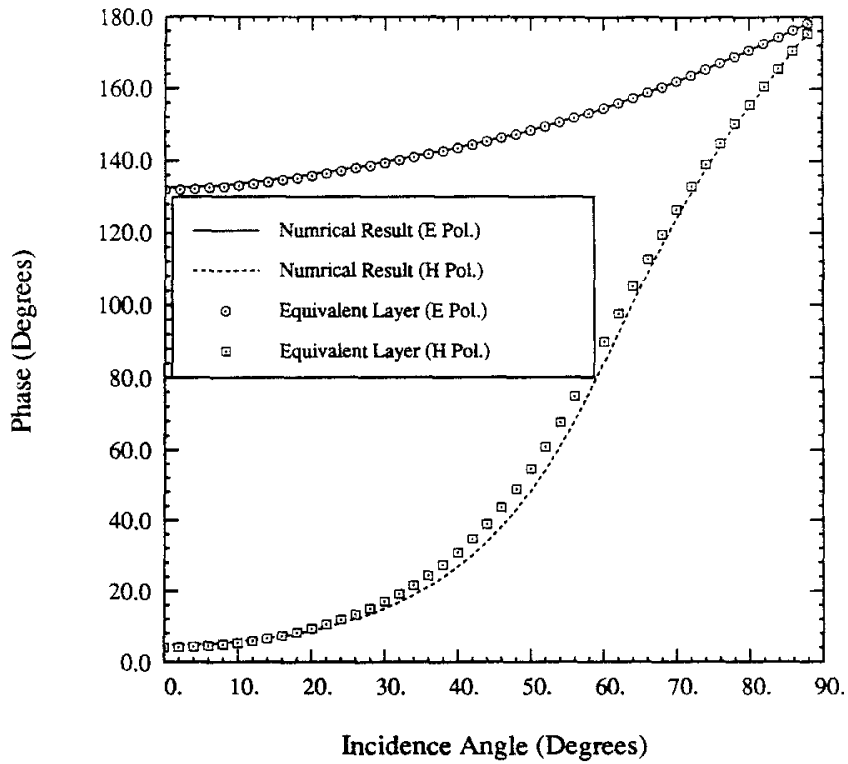

Figure 12: Phase of reflection coefficient of a corrugated surface for both $\mathrm{E}$ and $\mathrm{H}$ polarizations versus incidence angle; $L=0.25 \lambda_{0}$. 


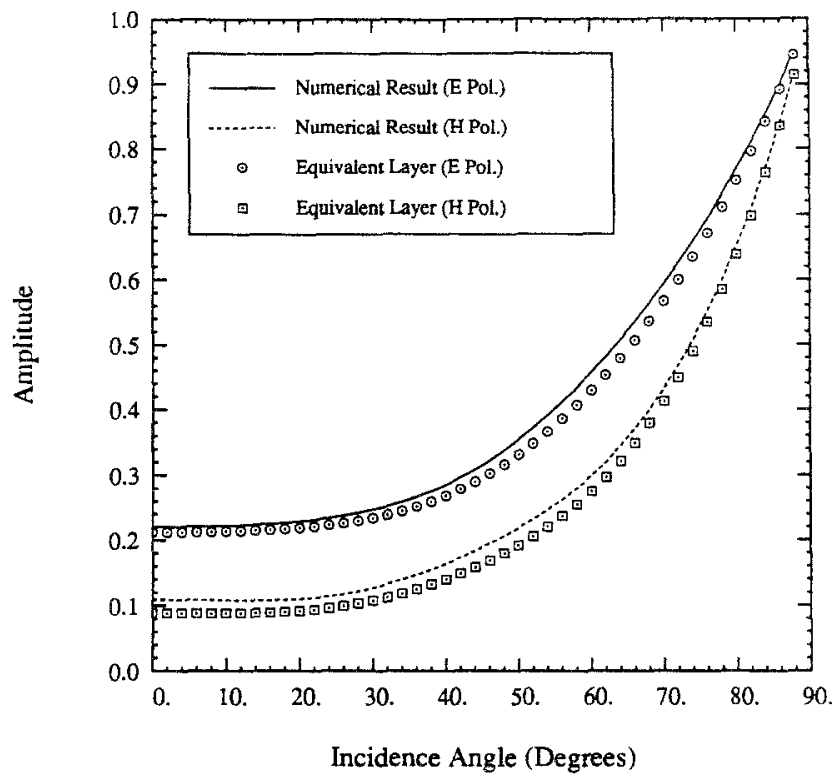

Figure 13: Amplitude of reflection coefficient of a corrugated surface for both $\mathrm{E}$ and $\mathrm{H}$ polarizations versus incidence angle; $L=0.4 \lambda_{0}$.

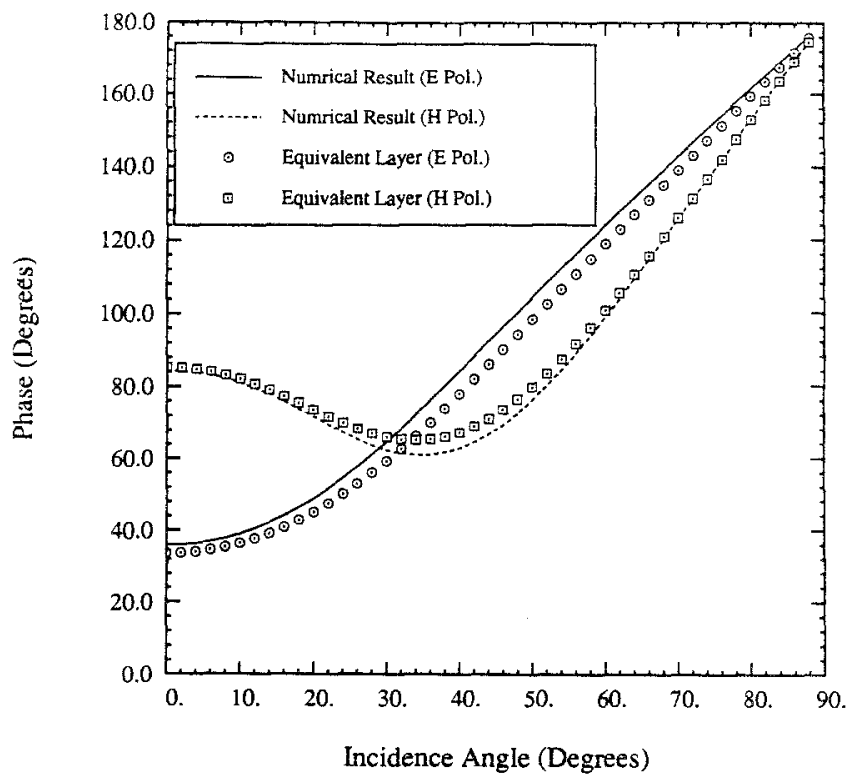

Figure 14: Phase of reflection coefficient of a corrugated surface for both $\mathrm{E}$ and $\mathrm{H}$ polarizations versus incidence angle; $L=0.4 \lambda_{0}$. 

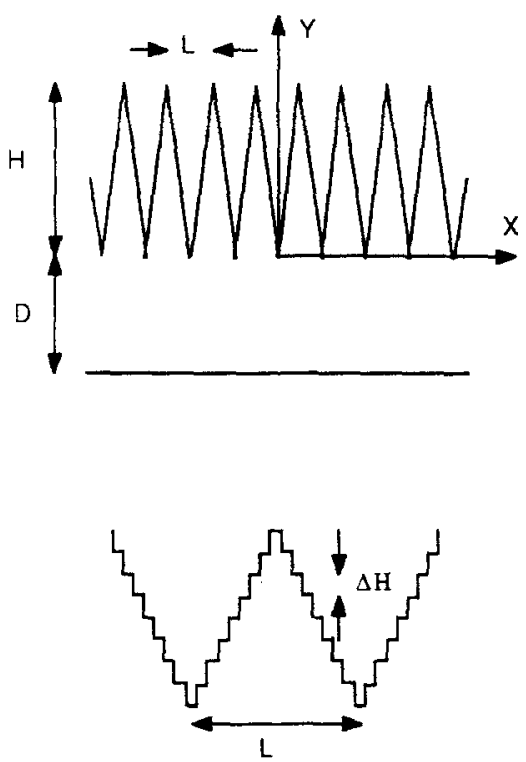

Figure 15: Geometry of a wedge-shape microwave absorber and its staircase approximation.

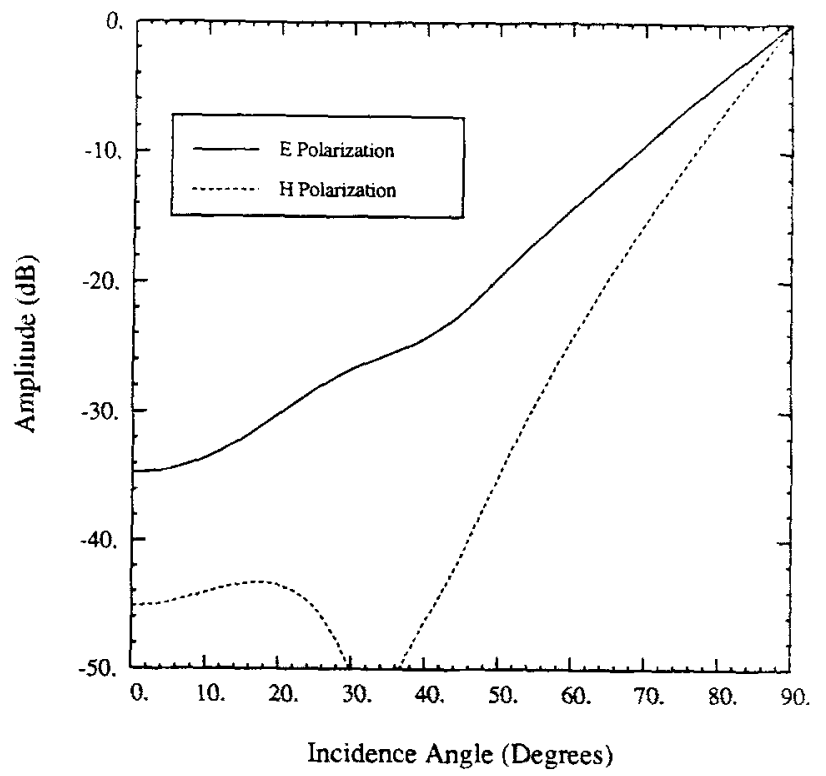

Figure 16: Amplitude of reflection coefficient of a werge-shape microwave absorber for both $\mathrm{E}$ and $\mathrm{H}$ polariżations versus inciclence angle; $L=0.4 \lambda_{0}, H=1.5 \lambda_{0}, D=1 \lambda_{0}$, and $\epsilon=2.5+\dot{8} 0.5$. 
shown that the equivalent medium is uniaxial. The equivalent tensor elements are obtained by finding the zeroes of the transcedental equations and at low frequencies analytical expressions are derived. The validity of this technique is verified by comparing the reflection coefficient of the equivalent layer with that of the corresponding corrugated surface using the moment method. Excellent agreement was obtained for values of $L<\lambda_{0} / 2$. Although the analysis is carried out for infinite slabs, it is shown that the effects of the discontinuities can be neglected. Using a multilayer approach the applicability of the technique is extended to handle periodic surfaces with arbitrary cross sections which can be used to analyze millimeter wave absorbers and dielectric plate polarizers.

\section{Acknowledgement}

This work was supported by NASA under contract NAGW 1334.

\section{References}

[1] Bodnar, D.G, and H.L. Bassett, "Analysis of an anisotropic dielectric Radome," IEEE Trans. Antennas Propag., pp. 841-846, Nov. 1975.

[2] Kong ,J.A., Electromagnetic Wave Theory, New York: John Wiley \& Sons, 1985.

[3] Morita, T., and S.B. Cohen, "Microwave lense matching by simulated quarter-wave transformers," IRE Trans. Antennas Propag., pp.33-39, Jan. 1956.

[4] Padman, R. "Reflection and cross-polarization properties of grooved dielectric panels," IEEE Trans. Antennas Propag., 26, pp. 741-743, 1978.

[5] Sarabandi, K., "Study of periodic dielectric surfaces: Simulation with anisotropic layers and application to high frequency scattering from corrugated convex bodies.", Radiation Laboratory Report No. 024557-2-T, The University of Michigan, February 1990. 